\title{
Data encryption standard based protection method of privacy data on Internet of Things
}

\author{
Xiaohui CHENG ${ }^{1, a}$, Zhongning LIANG ${ }^{1, b}$ \\ ${ }^{1}$ College of Information Science and Engineering, Guilin University of Technology, Guilin \\ 541006,Guangxi,China \\ a cxiaohui@glut.edu.cn, b similartochris@qq.com
}

Keywords: Internet of things; privacy data protection; data encryption standard; information security

\begin{abstract}
There are a lot of information and network security threats along with the rapid development of Internet of things (IoT). This paper according to the environmental features of the IOT node limited hardware resource and its requirements, and takes aim at the privacy information security issues for the IoT, an encryption method of lightweight data encryption is designed based on data encryption standard (DES). In order to reduce the complexity of the algorithm but ensuring the security, this algorithm simplified the data packet and the iterative rounds; at the same time added the random selection of S boxes, and improved sub key generation scheme to achieve some degree of "different keys in each encryption". Experiments show that, the algorithm reduces the computational complexity and resource cost, improve operation efficiency. It is a suitable method of privacy data protection in the IoT.
\end{abstract}

\section{Introduction}

The development of Internet of things has received a lot of attention, and it even rises to the height of the national strategy. The application of the Internet of things is very extensive. Specifically, it can be applied to many fields such as the military, industrial workshop, agricultural monitoring, public security, health care, smart home, and environmental monitoring. The development of the Internet of things is considered to be the third wave of the information industry following the computer, Internet and mobile communication network [1]. However, we must pay attention to the security threat of information and network of the Internet of things at the same time when Internet of things promotes the convenience of living to us. Transfer of information of Internet of things mostly by means of wireless, which give many criminals a channel to obtain improper benefits. Therefore, the security problem of Internet of things and information has become an important factor which can restrict the widespread of Internet of things. And the protection of data and privacy is the priority among priorities.

Many of the applications of the Internet of things are closely related with people's life [2]. The private data and privacy of which directly in relation to the people (such as Personal home address, the ECG data of medical equipment, food processing formula etc.) need to protect urgently. It is an effective and generic approach to protect the data by data encryption in the process of data transmission. And this method can avoid the defects of non-compatibility [3]. However, because of the small storage space, the weak processing ability and the limited hardware resources of the perception layer terminal of Internet of things, many of the traditional encryption algorithms cannot be used directly before the transmission of data of Internet of things. Therefore, the corresponding encryption algorithm is designed by the characteristics of Internet of things perception layer.

In this paper, in order to achieve the requirements of fast encryption speed, low computational complexity, less resource consumption, safety and security when processing a small amount of data. We combine the traditional DES algorithm and the characteristic of perception layer of Internet of things and then design a lightweight encryption algorithm. In the aspect of control algorithm complexity, we cut down the length of the encrypted data packet, the key length and the number of iterations for encryption of DES. In order to increase the security of the algorithm, we add a random 
selection method when selecting the $\mathrm{S}$ box. And make logic operation by the left half of encryption and the sub key and then enter the next step. Finally, we realized the design algorithm, and analyze its overall performance.

\section{Protection of Privacy Data in Internet of Things}

In recent years, there have been some researchers make research of security threats and the problem of privacy and data protection of Internet of things [3, 4], and discussed the research direction in the future of privacy protection of Internet of things [5]. This paper takes aim at the protection of the privacy of data and achieves privacy protection by the use of encryption technology. There are many demand services, perceptual information and interactive data need to be processed in the perception layer of Internet of things. And it will generate a large amount of private data, Such as the home address, tourist routes, such as the license plate number which are released by the social applications. This private data may face with eavesdropping attacks, replay attack, and the distorting attacks by criminals if the information directly exposed in the wireless network transmission [6]. Data encrypting can achieve data protection in the process of transferring data.

Because of the various types and the different function of Internet of things enabled devices, it will generate a large amount of data which will bring great workload. Our algorithm password-protects the location information and identity information and other private data of users so that it can reduce the processing burden of nodes of Internet of things. And improve the efficiency of data protection. And it can play the advantages of lightweight encryption method and ensure the safety of data at the same time.

The selective lightweight data encryption is a suitable method for privacy data preserving of Internet of things. It has a higher degree of privacy protection compared with the method of routing protocol and the Anonymization method. But its disadvantage is the long delay calculation and the high system resource consumption; these shortcomings are sensitive in the limited sensing nodes of Internet of things. It is a research direction of data privacy protection of Internet of things of how to reduce the complexity of encryption algorithm effectively and reduce the computational time and resource consumption to adapt to the demands of the environment of Internet of things. The simple schematic diagram of data privacy protection is shown in Fig1.

\begin{tabular}{|c|c|c|c|c|c|c|}
\hline \multirow{2}{*}{$\begin{array}{c}\text { Before } \\
\text { protected: }\end{array}$} & Name & Sex & Origin & Age & Education & Address \\
\hline & Zhang San & Male & Wuhan Hubei & 31 & Bachelor & $\begin{array}{c}\text { Chaoyang District } \\
\text { Democratic Road No. } 23\end{array}$ \\
\hline \multirow{2}{*}{$\begin{array}{c}\text { After } \\
\text { protected: }\end{array}$} & Name & Sex & Origin & Age & Education & Address \\
\hline & Zhang San & Male & Wuhan Hubei & 31 & Bachelor & \\
\hline
\end{tabular}

Fig1. Simple schematic diagram of data privacy protection

\section{Design of Lightweight Data Encryption Method}

The traditional DES algorithm is a symmetric encryption algorithm; it can meet the security requirements of the aspect of data protection in Internet of things. The algorithm packets the encrypt plaintext for 64 bit, and its dense iterative in 16 round; the sub key of each round is 56 bit. In the limited resources environment of nodes of Internet of things, the DES algorithm runs slower and it occupies larger storage space, so DES is not applicable. In this paper, some improvement is made to the applicability according to the Internet of things' environment based on DES algorithm. 


\subsection{Design of the Encryption Process}

The purpose of the improved algorithm is to reduce the complexity and efficiency of the algorithm in the encryption process. In this algorithm, we halve the encrypted plaintext group, length of the encryption key and the rounds of encryption iterative. It means that the length of groups which is composed of the 0,1 character string of the plaintext $\mathrm{x}$ is $32 \mathrm{bit}$; the length of groups which is composed of the 0,1 character string of the key $\mathrm{k}$ is 28 bit; The iterative rounds for encryption is 8 . We cancel the initial permutation and its inverse permutation because it does not affect the encryption and it doesn't have cryptographic significance [8]. So we reduced the complexity of the algorithm greatly. The algorithm can obtain strong pseudo randomness after 4 rounds of iterative [3], and the algorithm will have an "avalanche effect" after 5 rounds of iterative [9], so we can guarantee the streamlined encryption algorithm. Assume that the plaintext $x=x_{1} X_{2} x \cdots X_{32}$, the encryption key $k=k_{1} k_{2} \cdots k_{28}$, the encryption process can be expressed as $D E S(x)=T_{8} \bullet T_{7} \bullet \cdots \bullet T_{2} \bullet T_{1}(x)$. The specific algorithm flow can be shown in Fig2.

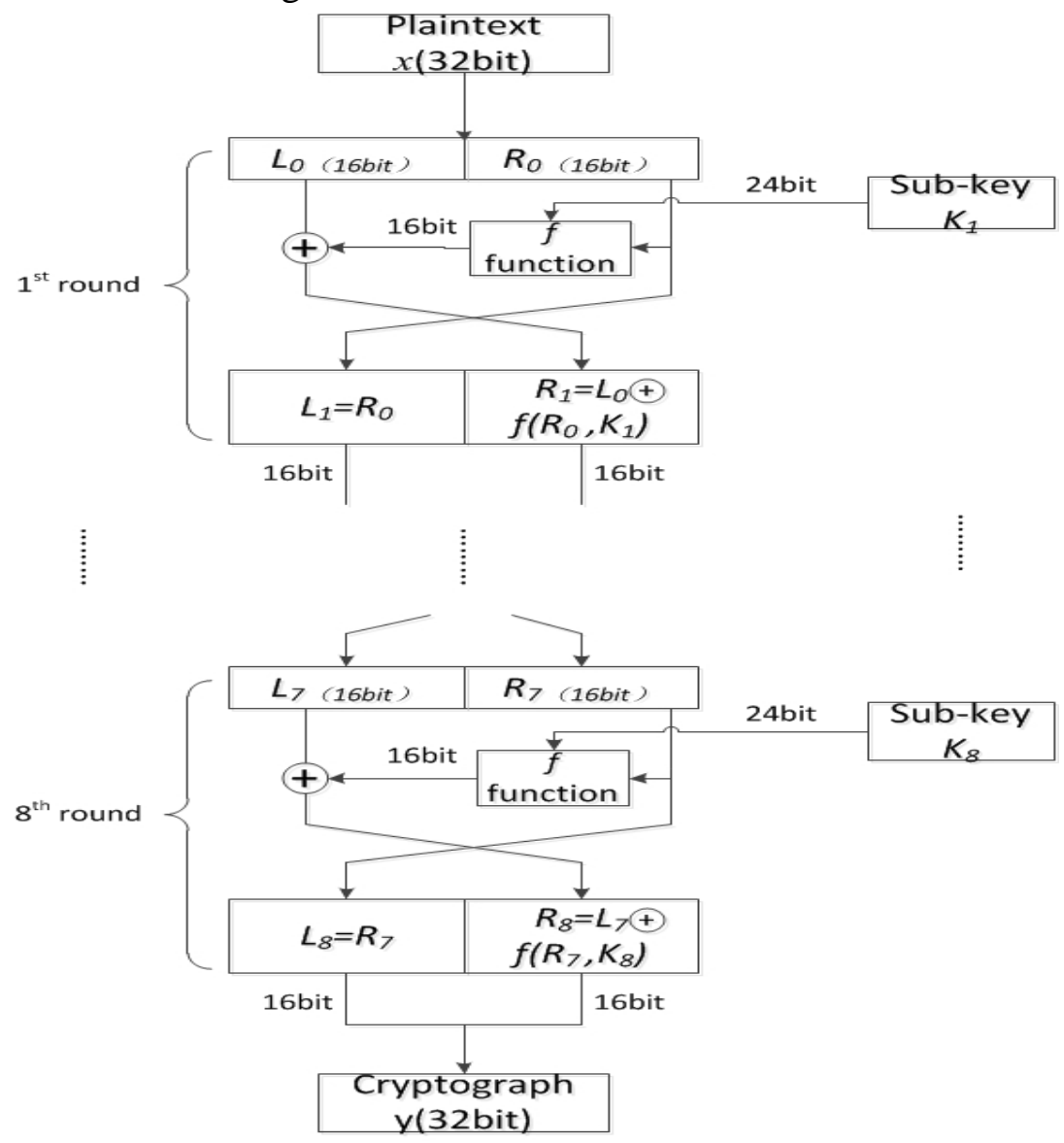

Fig2. Algorithm flow chart

Algorithm encryption process can be divided into plaintext initialize, sub-key generates and iterative process [10], S box selection scheme included in iterative process. Key generation scheme and $S$ box selection scheme will be described below in detail. The improvement of iterative process basically has the same process of DES, but simplify the rounds of iterative. Firstly, divided the encrypt plaintext $x$ into two equal length string $L_{0}, R_{0}$, the right piece of data $R_{0}$ expanded into 24 bit through $\mathrm{E}$ function, and reacts with corresponding sub key $\mathrm{K}_{\mathrm{i}}$ in $\mathrm{f}$ function, then through the $\mathrm{S}$ box become 16bit data, it is the output of $\mathrm{f}$ function. After that, the output of $\mathrm{f}$ function proceed bitwise XOR operation with the left piece of data $\mathrm{L}_{0}$ can be get the new output right piece of data. And the left piece of output data is equal to the input right piece of data. The iterative formula is $L_{i}=R_{i-1}, R_{i}=L_{i-1} \oplus f\left(R_{i-1}, K_{i}\right) ; i=1,2, \cdots, 8$. After eight iterations, the result is $L_{8} R_{8}$ switch left/right to get $\mathrm{R}_{8} \mathrm{~L}_{8}$, which is cryptograph $\mathrm{y}$.

The process of decryption in this algorithm almost equal to the process of encryption, just has different order in sub-key $\mathrm{K}_{\mathrm{i}}$, needed to used eight iterations of sub keys in reverse, that is, first round 
of decryption use $\mathrm{K}_{8}$, second round of decryption use $\mathrm{K}_{7}$, and so on. The decryption process can be expressed as: $\operatorname{DES}^{-1}(y)=T_{1} \bullet T_{2} \bullet \cdots \bullet T_{7} \bullet T_{8}(y)$.

\subsection{The S Box Design}

The purpose of $\mathrm{S}$ box and below the key generation scheme design and improvement is to enhance the strength of algorithm encryption and the degree of safety, meanwhile, saving running cost of the algorithm and increasing execution speed. A kind of regard time as the S box's random number sequence generate methods is presented by the scholars, to implement the different plaintext group generate the different cipher, that is, "different keys in each encryption", which improve the DES block cipher's attack strength. But this method has high algorithm complexity, doesn't match the idea of this paper.

This article directly using randomize three number of 0,1 string to confirm the selection and order of $8 \mathrm{~S}$ boxes in original DES. Meanwhile, have no effect on algorithm complexity when enhance algorithm strength. The improved f function's internal process is shown in Fig3.

Definition 1: Random function: Randomize three number of 0, 1 string; determine $S_{i}$ with its corresponding decimal number. $\mathrm{S}$ box: $\mathrm{S}$ box use DES to define two-dimensional tables, each box $\mathrm{S}_{\mathrm{i}}$ doesn't repeat $(0 \leq \mathrm{i} \leq 7)$.

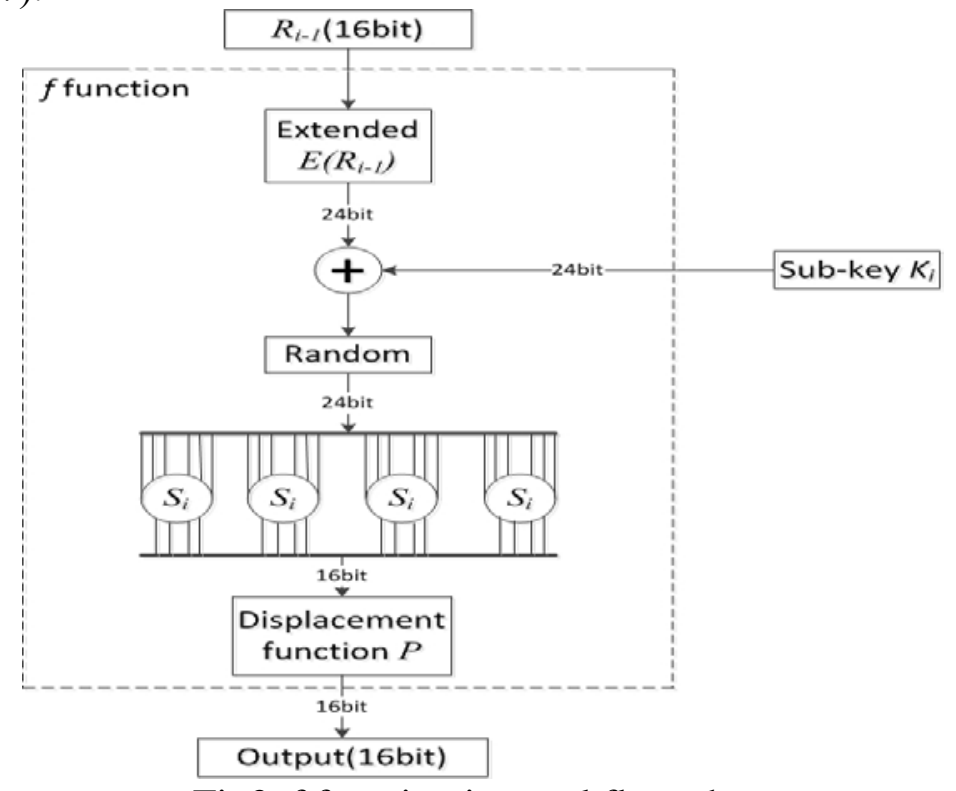

Fig3. f function internal flow chart

Every S box use 6 bit input to provide 4bit output. In the operation process, rower is consisted by the input data of the first and sixth binary characters, while column is consisted by the middle of the four binary characters, and through the row and column to oriental the elements need to turn into 4 bit binary number as output. Assumption 6 bit input is $\mathrm{A}=a_{1} a_{2} a_{3} a_{4} a_{5} a_{6}$, the corresponding row is $\mathrm{R}=a_{1} a_{6}$, the column is $\mathrm{C}=\mathrm{a}_{2} \mathrm{a}_{3} \mathrm{a}_{4} \mathrm{a}_{5}$, then in the Two-dimensional table Si can be query to the row $\mathrm{R}$, column $C$ correspond the element $B$, finally use 4 bit binary char to express $B=b_{1} b_{2} b_{3} b_{4}$ as $S$ box's output. Due to $a_{1} a_{2} a_{3} a_{4} a_{5} a_{6}=a_{1} a_{6} * 16+\mathrm{a}_{2} \mathrm{a}_{3} \mathrm{a}_{4} \mathrm{a}_{5}$, therefore, when using software is realized by algorithm, can line by line connected $S$ box 4 rows 16 columns of two-dimensional table, consisting of one dimensional table, changing the original $S$ box locate mode $S_{i}(R, C)$ into directly find a dimension table which corresponding $a_{1} a_{2} a_{3} a_{4} a_{5} a_{6}$ 's values. In this way, it can improve the running speed of the program to some extent.

\subsection{Sub Key Design}

Throughout the whole iterative process of DES algorithm, it can be see that in the process of encryption, after plaintext $\mathrm{x}$ is divided into left and right two parts, and expand the right part $\mathrm{R}_{\mathrm{i}-1}$ by $\mathrm{E}$ function then with the sub key to take part in the function, $f\left(R_{i-1}, K_{i}\right)$ and $\mathrm{L}_{\mathrm{i}-1}$ performs a bitwise exclusive XOR operation to get the new $R_{i}$. But the left part doesn't involve $f$ function or $S$ box changing, which bring algorithm certain security hidden danger [11]. So this article use the left part 16bit plaintext $\mathrm{L}_{\mathrm{i}-1}$ which also expend by $\mathrm{E}$ function, after that, the expended result will performs a 
bitwise exclusive AND operation with 24bit sub key $K_{i}$, then the new sub key will involve in the $f$ function. The improved algorithm of iterative process is shown in Fig4.

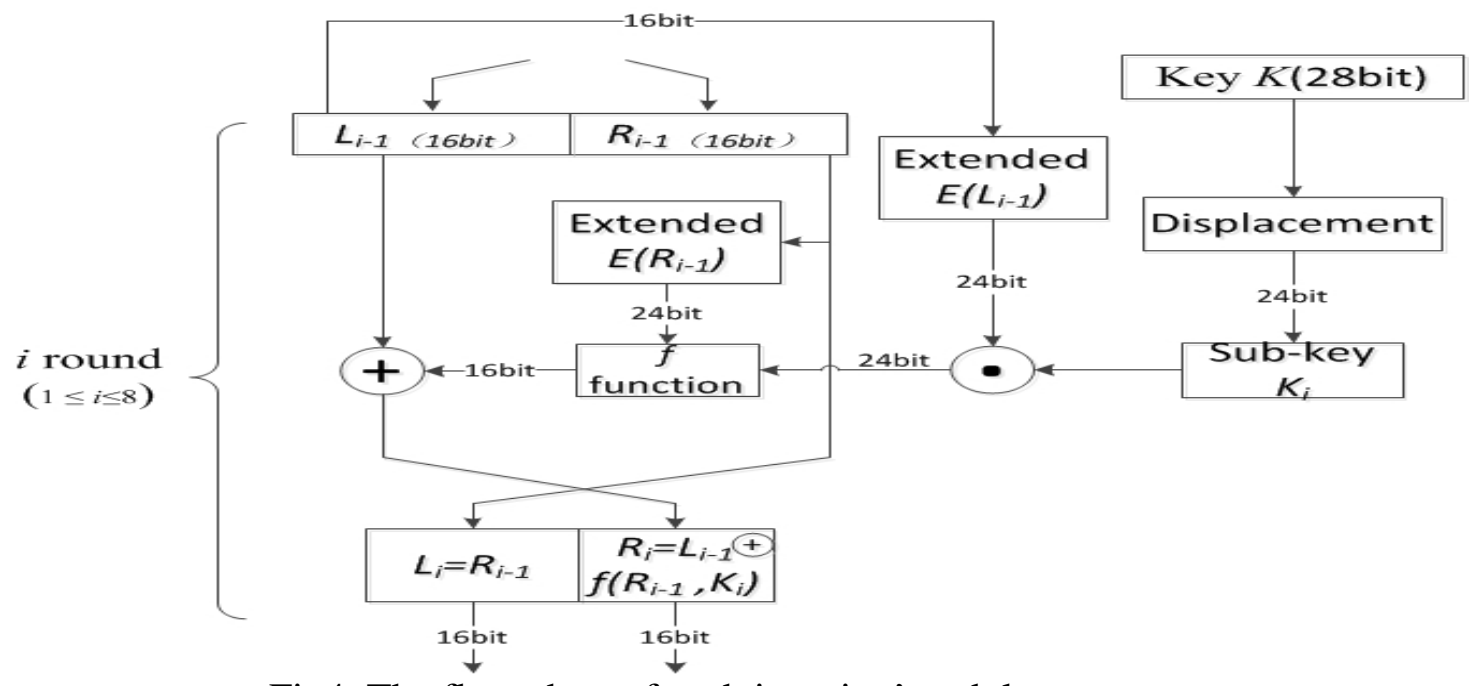

Fig4. The flow chart of each iteration's sub key generate

In the improved algorithm of iterative process, not only make the full use of the left part data $\mathrm{L}_{\mathrm{i}-1}$, but also get the plaintext involve in the generate sub key $\mathrm{K}_{\mathrm{i}}$ process, this scheme add the sub key selector mode, then use different $\mathrm{L}_{\mathrm{i}-1}$ each time, achieving “different keys in each encryption” to some extent, enhance the security of the algorithm as well. Relevantly, because of $\mathrm{L}_{\mathrm{i}-1}$ is divided in iterative process, expanded $\mathrm{E}$ function has already exist, so it does not need to take up extra design and resource-intensive; While the original sub key $\mathrm{K}_{\mathrm{i}}$ and $\mathrm{L}_{\mathrm{i}-1}$ will proceed AND operators by Bitwise, and it is simple in hardware to implement the logic operation $K_{i} \bullet L_{i-1}$. In a word, the improvement of the design can save a lot of additional cost of the system resources.

\section{Experiments and Analysis}

\subsection{Algorithm Experiment and Efficiency Analysis}

In order to better reflect the running speed and the consumption of system resources of the improved algorithm. In this paper, the elliptic curves cryptography (ECC) is added to compare, which is often used in the Internet of things. The improved algorithm is implemented in Java; the test encrypted data is string (five groups, 128bit), encryption and decryption 1000 times. The hardware environment of this experiment is: CPU: Intel(R) Core(TM)2 Duo T6570 @2.10GHZ; memory: 2GB; hard disk: 320GB; JDK version: 1.7.0_67; Test results are shown in Table1 and Fig5.

Table1. Encryption and decryption time-consuming of three algorithms

\begin{tabular}{ccccccc}
\hline Time & \multicolumn{2}{c}{ ECC } & \multicolumn{2}{c}{ DES } & \multicolumn{2}{c}{ Improved DES } \\
\cline { 2 - 7 } /s & $\begin{array}{c}\text { Encryp } \\
\mathrm{t}\end{array}$ & $\begin{array}{c}\text { Decryp } \\
\mathrm{t}\end{array}$ & $\begin{array}{c}\text { Encryp } \\
\mathrm{t}\end{array}$ & $\begin{array}{c}\text { Decryp } \\
\mathrm{t}\end{array}$ & $\begin{array}{c}\text { Encryp } \\
\mathrm{t}\end{array}$ & $\begin{array}{c}\text { Decryp } \\
\mathrm{t}\end{array}$ \\
\hline Group 1 & 43.34 & 45.63 & 4.53 & 4.81 & 2.78 & 3.46 \\
\hline Group 2 & 40.57 & 46.89 & 4.40 & 4.98 & 2.97 & 3.40 \\
\hline Group 3 & 41.36 & 45.90 & 4.54 & 4.97 & 2.81 & 3.51 \\
\hline Group 4 & 41.87 & 45.60 & 4.31 & 4.85 & 3.06 & 3.32 \\
\hline Group 5 & 40.36 & 46.06 & 4.58 & 4.92 & 2.93 & 3.28 \\
\hline Average & 41.50 & 46.02 & 4.47 & 4.91 & 2.91 & 3.39 \\
\hline
\end{tabular}



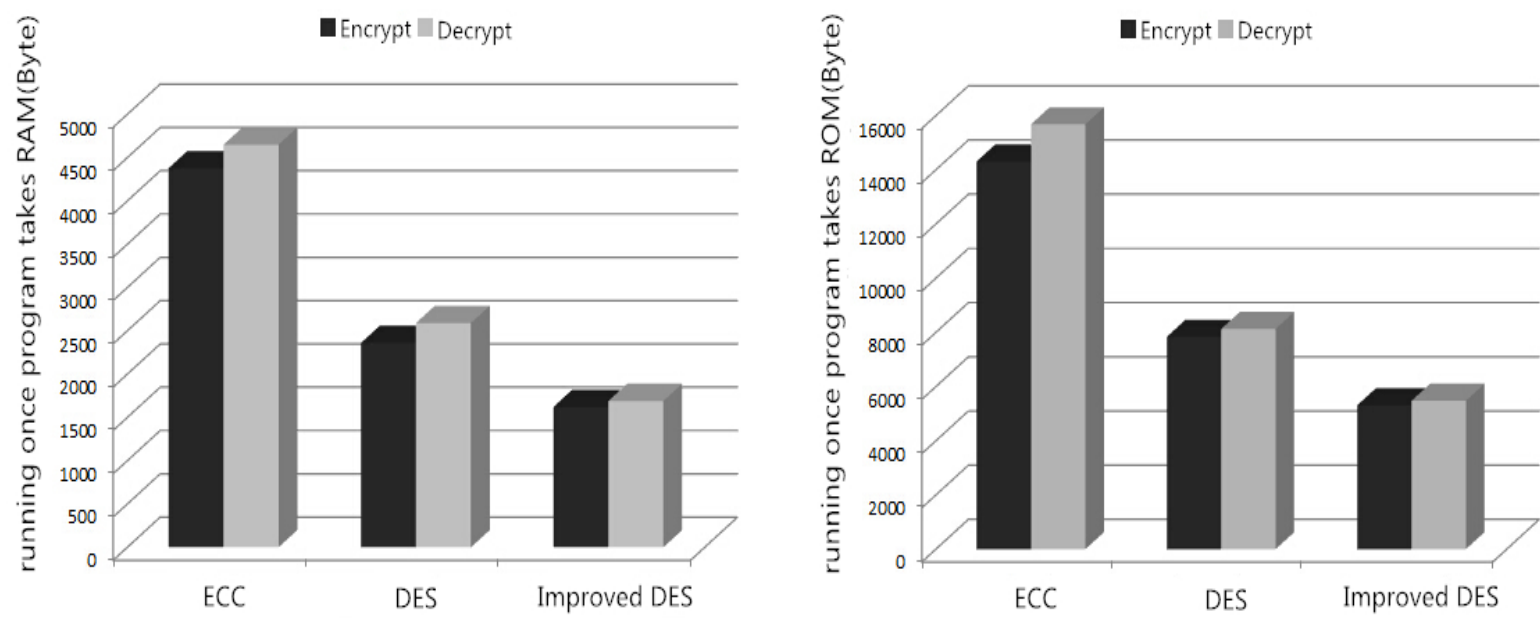

Fig5. Encryption and decryption takes up storage space of three algorithms

Form table1, we can see that the improved algorithm is less time-consuming than the original DES algorithm, which reduces the time of the encryption by 34\%, and reduces the decryption time by $31 \%$, since the improved algorithm after streamlining the number of iterations and some of the shift operation. Efficiency is far higher than the common ECC algorithm in the Internet of things. It can be seen that this algorithm has some advantages in the protection of small amount of data encryption, to ensure the implementation of better efficiency of the privacy data protection in IoT. From Figure5, can be seen directly, three algorithms to complete once encryption and decryption takes RAM and ROM within $20 \mathrm{~KB}$, the lightweight feature of the algorithm is presented. Among them, ECC takes up most of the storage space; it is also related to the key length. DES algorithm is second, and the system resource consumption of the improved algorithm is about $2 / 3$ of DES, it is more suitable for the limited system resource node of the IoT.

\subsection{Performance Analysis of the Algorithm}

1). Applicability. The perception notes of the Internet of things are less storage space and less processing power, the amount of data privacy protection is also smaller. The improved encryption method has the lightweight property, Low complexity, fast operation and less resources consumption, outstanding performance in a small amount of data processing. Suitable for sensor nodes in IoT and some systems which is low cost of communication, extremely limited hardware resources.

2). Security. The improved algorithm although reduced the number of rounds during the iterative process, but the condition of the "avalanche effect" is reached, that is ensure by the extended diffusion, $\mathrm{S}$ boxes and the replacement $\mathrm{P}$. It also has some random properties. The random selection of $\mathrm{S}$ box also brings some randomness to the algorithm. The data to be encrypted is added in the sub key generation scheme to achieve some degree of "different keys in each encryption”. The mentioned above are provided good security for the improved encryption algorithm.

\section{Conclusion}

Lightweight data encryption technology is a kind of ideal privacy data protection method in the environment of Internet of things. In this paper, the problem of information security for the Internet of things, combining the characteristics of the Internet of things and the requirements, a lightweight encryption algorithm based on DES is designed. With the simplification and optimization of encryption process, improvement of S box selection and sub key generation scheme, the algorithm has a good performance in the speed of encryption and decryption and system resource occupation. It is suitable for sensor nodes in IoT, and has good safety performance. Provided a valid method for the Internet of things privacy data protection. 


\section{Acknowledgements}

As the research of the thesis is sponsored by National Natural Science Foundation of China (No: 61262075), major scientific research project of Guangxi higher education (No: 201201ZD012) and Project Foundation of Guangxi Experiment Center of Information Sciencep (No: 20130206), we would like to extend our sincere gratitude to them.

\section{References}

[1] Floerkemeier C. Langheinrich M, Fleisch E, Mattern F. The Internet of Things[C]: Lecture Notes in Computer Science. Springer, 2008, 49-52.

[2] ATZORI L, IERA A, MORABITO G. The Internet of things: a survey [J]. Computer Networks, 2010,54(15) : 2787-2805.

[3] Changli Zhou. Protection strategy of privacy data for the Internet of Things[D]. Harbin: Harbin Engineering University, 2012.

[4] WEBER R H. Internet of things: new security and privacy challenges [J].Computer Law \& Security Review, 2010,26(1) : 23-30.

[5] Ping Qian, Meng Wu. Survey on privacy preservation in IoT[J]. Application Research of Computers, 2013,30(1) : 13-20.

[6] Junsong Zhang.Researches on key problems of security and privacy protection under Internet of Things[D]. Beijing: Beijing University of Posts and Telecommunications, 2014

[7] Limin Sun. Wireless sensor network [M]. Beijing: Tsinghua University press, 2005.

[8] Zhenyu Mei, Hong Zhang. Improvement for DES blocks cipher based on non S-box transformation [J]. Computer Engineering and Design, 2008, 28(17):4119-4121.

[9] Paar C, Pelzl J. Understanding cryptography: a textbook for students and practitioners [M]. Springer Science \& Business Media, 2009.

[10]Yuechao Hui, Yiming Wang. Design of lightweight encryption algorithm based on optimized S-box [J]. Communications Technology, 2010,05(48): 103-108.

[11]Liu Xiaoxing, Hu Changxia, Liu Mingsheng. The analysis and improvement of DES encryption algorithm[J]. Micro Computer Information, 2002, 22(4): 32-33.

[12]Xiangchao Fang, Xingbao Li. Optimization of data encryption based on a Key [J]. Computer Systems \& Applications, 2013, 22(9): 136-138.

[13]Anping Lu, Jimin Yang, Li Feng. A comparative study of several lightweight encryption algorithms[J]. Modern Electronics Technique 2014, 37(12) 37-41. 